\title{
Multi-Wavelength Photopolymerization of Stable Poly(Catecholamines)-DNA Origami Nanostructures
}

\author{
Pia Winterwerber, ${ }^{[a]}$ Colette J. Whitfield, ${ }^{[a]}$ David Y. W. Ng, ${ }^{*[a]}$ Tanja Weil ${ }^{*[a]}$ \\ [a] Pia Winterwerber, Dr. Colette J. Whitfield, Dr. David Y. W. Ng, Prof. Dr. Tanja Weil \\ Max Planck Institute for Polymer Research \\ Ackermannweg 10, 55128 Mainz (Germany) \\ E-mail: david.ng@mpip-mainz.mpg.de \\ weil@mpip-mainz.mpg.de
}

\begin{abstract}
Orthogonality is pivotal yet chemically challenging in the bottom-up synthesis of multicomponent nanostructures. Here, we leverage the fidelity of the DNA origami technique to install a multi-wavelength responsive photopolymerization system with nanometer resolution. By precisely immobilizing various photosensitizers on the origami template, which are only activated at their respective peak wavelength, we can control sequential polymerization processes. In particular, the triggered photosensitizers generate reactive oxygen species that in turn initiate the polymerization of the catecholamines dopamine and norepinephrine. We imprint polymeric layers at designated positions on DNA origami, which modifies the polyanionic nature of the DNA objects, thus, promoting their uptake into living cells while preserving their integrity. Our herein proposed methodology provides a rapid platform to access complex 3D nanostructures by customizing material and biological interfaces.
\end{abstract}

Spatial control and engineering of objects at nanometer resolution is imperative to the miniaturization of smart materials and devices. In both materials science and biomedicine, the demand for tools to construct multicomponent substructures across 3D space is required to expand the understanding of how surface patterns and object contours modulate interfacial forces. However, the construction of nanostructured surfaces on soft materials that are freely customizable is a bottleneck due to the lack of tools to precisely design them. This problem is further amplified for patterns that are much smaller $(<10 \mathrm{~nm})$ than the wavelength of light, where top-down approaches, such as lithography, reaches its limits. At this length scale, bottom-up approaches based on self-assembly provide the natural complementarity to top-down strategies in the fabrication of patterned soft materials.

Unlike the limitations posed by other systems based on synthetic polymers or peptides, DNA nanotechnology is equipped with the precision necessary to program nanostructured surfaces. ${ }^{[1]}$ Coupled with a DNA origami design, ${ }^{[2]}$ concepts to investigate distances between epitopes, ${ }^{[3]}$ protein assemblies, ${ }^{[4]}$ plasmonic devices, ${ }^{[5]}$ and biosensing ${ }^{[6]}$ have recently resulted in critical findings in nanomedicine and biophysics. In polymer chemistry and patterning, advances in radical and oxidative polymerization as well as polymer routing have demonstrated that the stringent conditions necessary for DNA origami can be made accessible to largely organic compounds. ${ }^{[7]}$ Conversely, the combination of DNA nanostructures with charged molecules and polymers has shown increased stability in physiological conditions and even in organic solvents, which have been crucial in the rapid expansion of the DNA origami platform in recent years. ${ }^{[8]}$ However, in comparison to the application driven counterparts, polymers on DNA origami have yet to show their synthetic potential beyond structured positioning by DNA hybridization on the template..$^{[9]}$

In this study, we control a series of photopolymerization reactions using orthogonal wavelengths to guide independent polymer patterns and fabricate layered structures on the DNA origami (Figure 1). Previously, we have shown that dopamine (DA) can be photopolymerized by protoporphyrin IX (PPIX) that is intercalated into a DNA G-quadruplex (G4), which allows polydopamine to form at designated positions pre-occupied by the G4.[10] Herein, we establish the wavelength orthogonality by using G4s containing eosin $\mathrm{Y}(\mathrm{EY})$ and methylene blue $(\mathrm{MB})$, which are activated in green $(525 \mathrm{~nm})$ and red $(625$ $\mathrm{nm}$ ) light, respectively. Together with blue light triggered PPIX $(410 \mathrm{~nm})$, these three catalyst centers 
produce reactive oxygen species (ROS) at their peak wavelengths to initiate the polymerization of catecholamines. By using both dopamine and norepinephrine (NE), we demonstrate that both monomers can be polymerized sequentially in different configurations. The formation of a polymer layer reduces the polyanionic nature of the DNA origami and can thereby facilitate its uptake into living cells, which can be imaged by fluorescence colocalization. Our approach will enable rapid and facile synthesis of multicomponent polymeric patterns on DNA origami with precise shapes and dimensions. Customizing the nano-biointerphase of DNA objects through surface modulation is crucial for various applications, e.g., cellular uptake for therapeutic delivery.

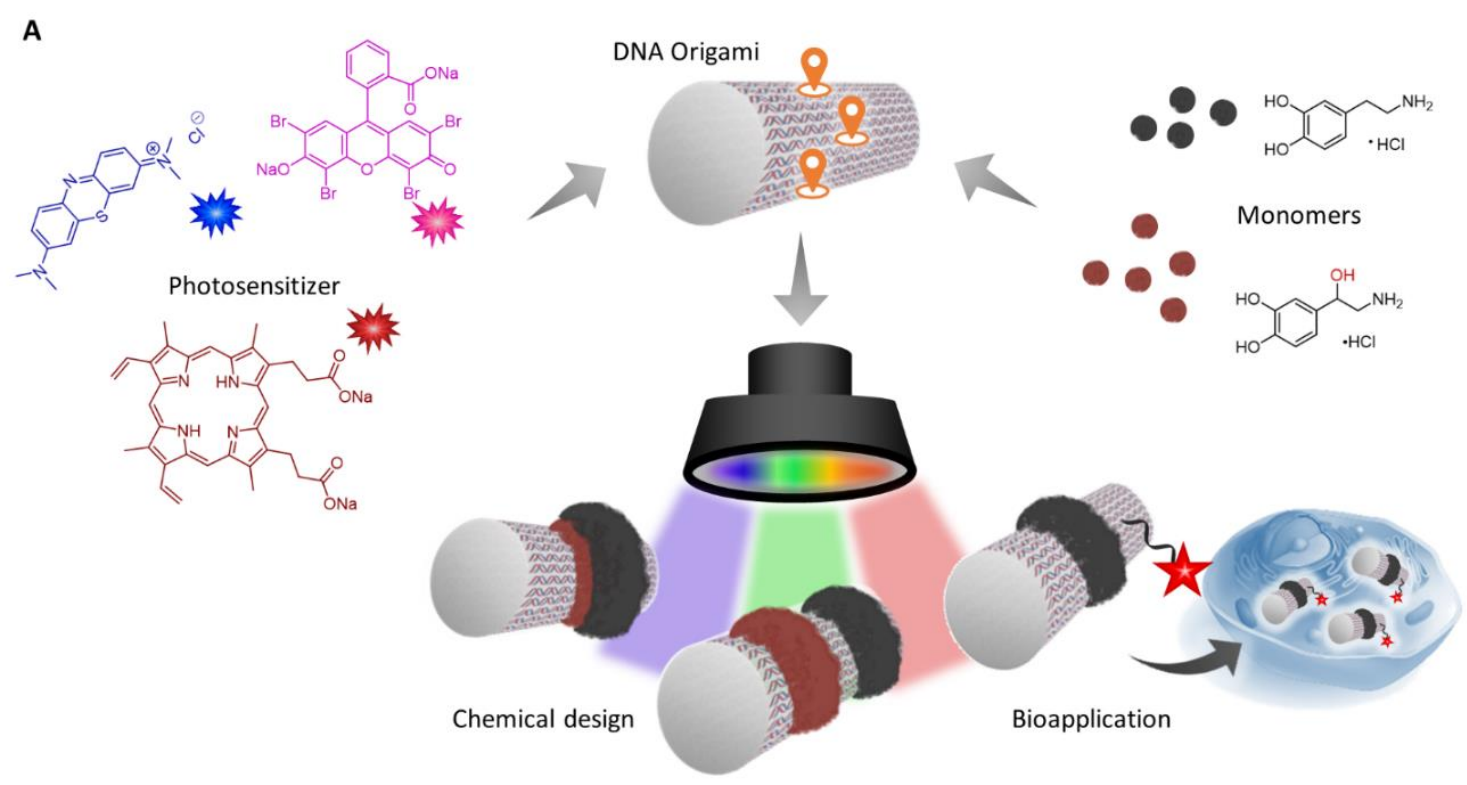

B

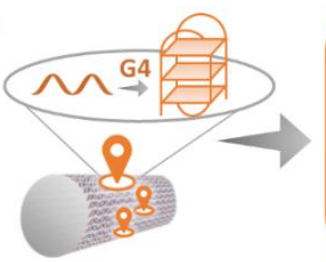

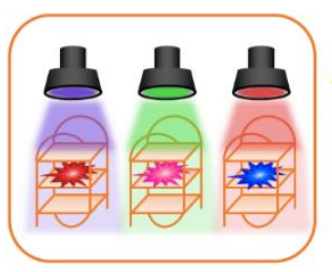

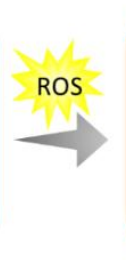

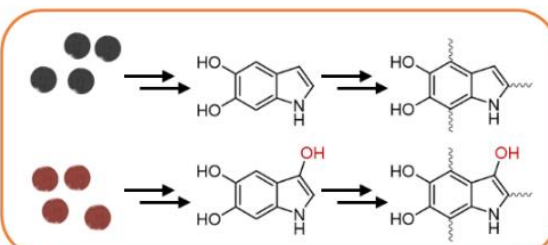

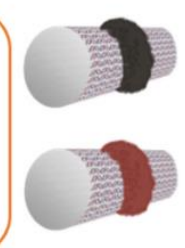

Figure 1 (A) Multi-wavelength photopolymerization on DNA origami tubes can be accomplished through the combination of various photosensitizers and two different catecholamine monomers. In this way, polymers can be imprinted at specific sites on the DNA templates under temporal and spatial control. Polymer-DNA hybrid structures can be further leveraged to modulate interactions at the cellular interface. (B) The reaction centers, consisting of G-quadruplex (G4) structures and photosensitizers, produce reactive oxygen species (ROS) at their peak wavelength to initiate polymerization. Mechanism and structure of both polydopamine $e^{[11]}$ and norepinephrine ${ }^{[12]}$ are still under elucidation. For reason of clarity only a few representative structures are depicted. Further information is provided in the supporting information (SI Figure 1).

To prepare 3D DNA origami tubes for photopolymerization, G-quadruplex structures were allocated at the surface in distinct patterns. These catalytic centers can be tuned for wavelength selectivity by nominating the photosensitizer that will sit within the G-quadruplex (5'-GGG TA GGG C GGG TT GGG-3'). As previously reported, the PPIX-G4 complex produces ROS under white light irradiation which in turn trigger oxidation and polymerization of dopamine. To suppress the well-known self-polymerization of dopamine and to control polymer formation, it is crucial to work in a slightly acidic environment ( $\mathrm{pH}$ 6.5). Herein, we found that only the blue light component $(410 \mathrm{~nm})$ possesses sufficient energy to initiate the polymerization of dopamine (SI Figure 2). Excitation at the Q-bands of PPIX-G4 in the visible spectrum produced insufficient oxidized dopamine species to fuel polymerization. Wavelength specificity in the green and red region was accomplished by hosting $E Y^{[13]}$ and $\mathrm{MB}^{[14]}$, respectively, within the G4 motif. The propensity of each catalyst to generate singlet oxygen $\left({ }^{1} \mathrm{O}_{2}\right)$ was analyzed using an assay based on imidazole and p-nitrosodimethylaniline (RNO). ${ }^{[15]}$ Both MB and EY demonstrated higher efficiencies in the production of ${ }^{1} \mathrm{O}_{2}$ than PPIX, as reflected by 7 and 11 times 
faster bleaching of RNO, respectively (SI Figure 3). The initiation of polymerization using EY-G4 and MB-G4 on a tube DNA origami was subsequently attempted at their respective wavelengths (EY: 525 $\mathrm{nm}, \mathrm{MB}: 625 \mathrm{~nm}$ ). The tube DNA origami scaffold was designed with a central ring containing photosensitizer-loaded G4 sequences. Using $10 \mathrm{~mm}$ of dopamine in $100 \mathrm{~mm}$ buffer (pH 6.5), UV/Vis spectroscopy showed successful polymerization into polydopamine (pDA) after $3 \mathrm{~h}$ (SI Figure 4A). Formation of intermediates including dopaminochrome $(320 \mathrm{~nm})$, oxidized oligomers $(480 \mathrm{~nm})$ and the eventual pDA $(700 \mathrm{~nm})$ could be monitored via their characteristic absorbances. ${ }^{[7 \mathrm{c}]}$ Spatial control over polymerization and the resulting nanostructure was verified by atomic force microscopy (AFM) (SI Figure 4B). A ring of polymers was successfully constructed where the patterned G4 sequences were installed, demonstrating that the change of catalytic centers and excitation wavelengths did not affect the control over the polymerization reaction. In comparison, the reaction kinetics of the oxidative polymerization demonstrated that the generation of each intermediate (dopaminochrome, oligomers etc.) including pDA was more efficient for EY (SI Figure 4C). Although the topological height profile analysis via AFM did not show significant height differences (SI Figure 4D), the polymer-ringed DNA origami tubes tended to aggregate due to the strong adhesiveness of pDA (SI Figure 5). Therefore, norepinephrine was introduced as a dopamine analogue to achieve well-dispersible nanoobjects that also remain stable in complex media without aggregate formation. Norepinephrine also belongs to the catecholamine family and poly(norepinephrine) ( $\mathrm{pNE}$ ) reveals material-independent modification capabilities similar to $\mathrm{pDA}$ but with an ultrasmooth surface morphology. ${ }^{[12 c, 16]}$ Chemically, NE possesses an additional hydroxyl group and this increase in hydrophilic interactions could potentially prevent aggregation of the formed nanostructures. In contrast to pDA, polymerization to pNE using all three photosensitizers showed a strong preference toward EY and MB (SI Figure 6). Monitored by UV/Vis spectroscopy, these observations suggested that oxidation of pNE requires a higher performance photosensitizer to fuel the polymerization reaction. Likewise, the eventual formation of pNE on the DNA origami showed that the polymerization was more efficient with $\mathrm{EY}$ and $\mathrm{MB}$ as the photosensitizer.

Based on the acquired reaction conditions and wavelength selectivities pertaining to DA and $\mathrm{NE}$, sequential polymerization steps were performed to fabricate multicomponent nanostructures. The DNA origami tubes were loaded with MB-G4s, and NE and DA were sequentially polymerized at 625 $\mathrm{nm}$ for $2 \mathrm{~h}$ each (Figure 2A). Successful polymerization was detected for both illumination phases, giving the characteristic profiles for pNE and pDA (SI Figure 7). The first irradiation phase resulted in the formation of a pNE layer of $5.2 \mathrm{~nm} \pm 1.5 \mathrm{~nm}$ (Figure 2B). Thereafter, excess NE and oxidized side products were removed by spin filtration and replaced by DA. The second irradiation phase yielded the pDA layer that contributed an additional height increase of $4.3 \mathrm{~nm} \pm 1.8 \mathrm{~nm}$ (total height: $9.5 \mathrm{~nm}$ $\pm 1.8 \mathrm{~nm}$ ) (Figure 2B). The contributed height increase of each component correlates well to their individual single polymerizations. In order to demonstrate that the second irradiation phase had initiated the polymerization of DA and not existing pNE (or its adsorbed oligomers), a control experiment was performed without the addition of DA. In this case, no additional polymers were formed (SI Figure 8). The upper limit of the layered components is dictated by the access of monomers towards the catalytic centers. At approximately $10-15 \mathrm{~nm}$ in total height, the polymerization can no longer be guided by the photosensitizer-G4 complex. 
A

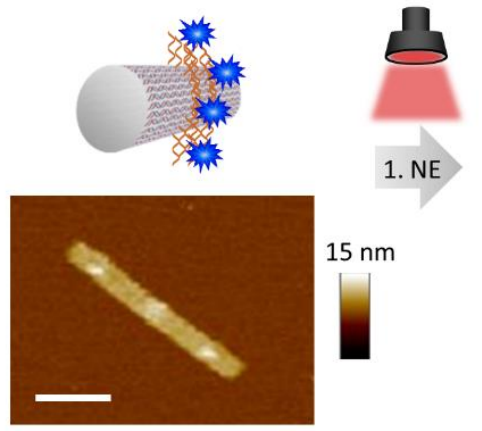

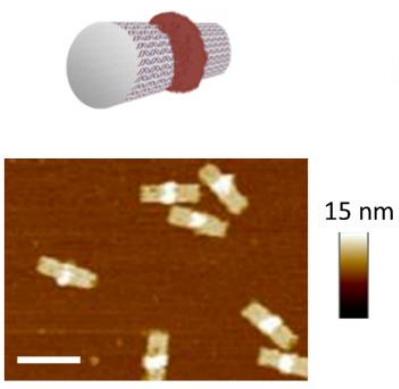
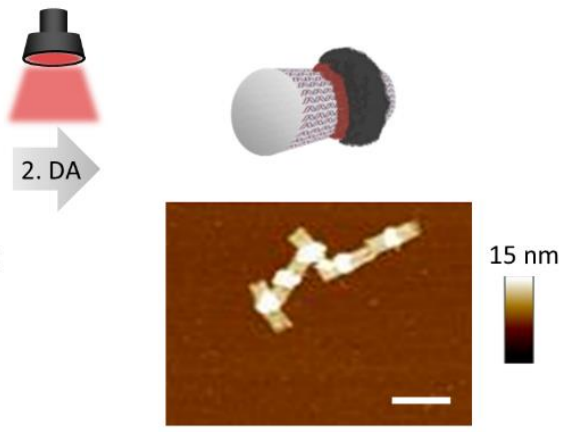

B
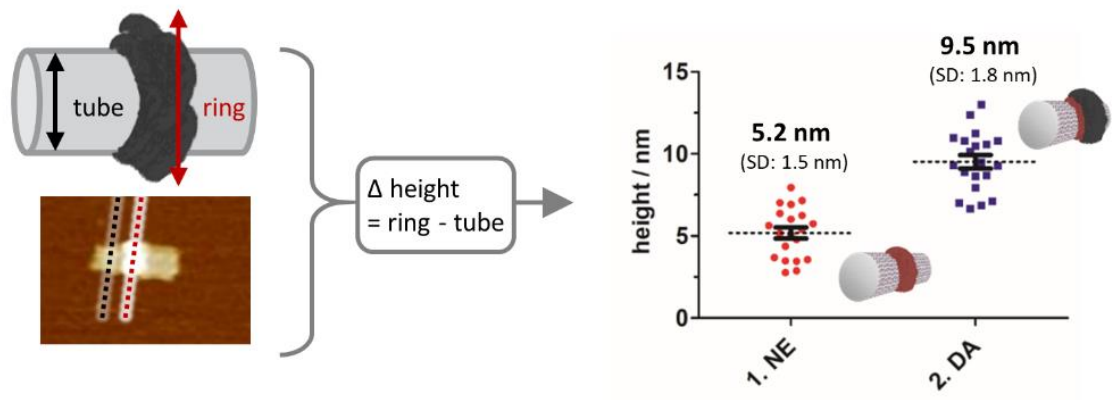

Figure 2 A two-step polymerization process demonstrates the potential of the origami system to induce a layer-by-layer formation of polymers. (A) DNA origami tubes are incubated with methylene blue as the photosensitizer and irradiated at $625 \mathrm{~nm}$. In the first illumination phase, norepinephrine (NE) is polymerized, followed by a purification step to remove remaining NE, and dopamine (DA) is added as the second monomer that also polymerizes under red light irradiation. AFM images show the topographical profile of the DNA origami tubes. The polymer is imprinted on top of the G4 patterns. Scale bars are $100 \mathrm{~nm}$. (B) To quantity the thickness of the polymer layers, $z$-value heights were recorded and calculated as depicted ( $n=20$; error bars are SEM).

Next, we demonstrated wavelength orthogonality for a two-step polymerization to achieve reaction selectivity. The dormancy of $\mathrm{MB}$ and $\mathrm{EY}$ at opposing wavelengths was first investigated. $\mathrm{MB}$ showed no generation of the oxidized species of DA when irradiated at $525 \mathrm{~nm}$, and, vice versa, EY was not active at $625 \mathrm{~nm}$ (SI Figure 9). To ensure that a consecutive activation of each photosensitizer can continuously trigger the polymerization of dopamine, the DNA origami tubes were loaded with ring patterns of EY-G4 and MB-G4 at each tubular end. Polymerization was initiated with DA (10 mM, pH 6.5) by sequential irradiation at $625 \mathrm{~nm}$ and $525 \mathrm{~nm}$ for $3 \mathrm{~h}$ each. The generation of oxidized intermediates and pDA was verified by UV/Vis spectroscopy (SI Figure 10A). Control experiments ascertain that the integrity of the DNA origami tube was not damaged by the prolonged irradiation and by the ROS produced by the photosensitizers (SI Figure 10B).

Subsequently, to broaden the approach, we decoupled the loading of both EY and MB photosensitizers into sequential steps to show that DNA hybridization in a post-polymerization fashion is robust and reliable (Figure 3). The first step involved the attachment of MB onto a DNA origami tube equipped with a single ring of G4 sequences. Irradiation at $625 \mathrm{~nm}$ for $2 \mathrm{~h}$ with $\mathrm{NE}(10 \mathrm{mM}, \mathrm{pH} 6.5)$ formed the first polymer ring, which could be visualized by AFM and UV/Vis spectroscopy (Figure 3B+C). Excess NE was removed by spin filtration and the second photosensitizer, EY-G4, was hybridized onto an opposing ring pattern of the same DNA tube using a temperature ramp. The second polymerization step was conducted at $525 \mathrm{~nm}$ for $2 \mathrm{~h}$ with $\mathrm{DA}(10 \mathrm{mM}, \mathrm{pH}$ 6.5) as the monomer to afford the final nanostructure where $\mathrm{pNE}$ and $\mathrm{pDA}$ each occupies a single ring. The oxidation profiles of both NE and DA in this dual component system showed consistent polymerization kinetics when compared to the single component system. Furthermore, by comparing the average polymer heights of each irradiation period, sequential ring formation can be tracked (Figure 3D). In the first step, only one position on the origami tube showed polymer formation $(4.8 \pm 0.9 \mathrm{~nm}$ ), which is also clearly depicted in the height difference of this polymer ring and the adjacent ring pattern ( $\Delta$ of $4.2 \pm 0.7 \mathrm{~nm}$ ). In the second step, a second polymer ring was grown at the designated position with a thickness reaching dimensions of the previously grown ring. Indeed, both polymers exhibit similar heights $(\Delta$ of $1.6 \pm 1.5 \mathrm{~nm}$ ). However, it is important to note that the possibility of activated species diffusing to the initial ring cannot be totally excluded even though the 
observable height contribution to the initial ring was minimal. The presence of all reactive components, i.e., monomers, patterned photosensitizers on the DNA origami and the light source, is essential for the formation of the nanostructure. Control experiments involving only monomer without irradiation, or without embedded photosensitizer did not show polymerization (SI Figure 11). In both coupled and decoupled methods, we demonstrate that access to sophisticated and multicomponent 3D DNA-polymer hybrids can be fabricated easily.

A

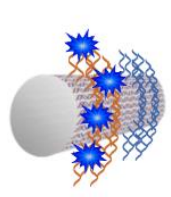

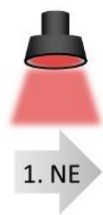

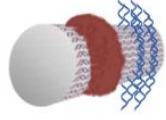

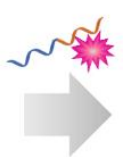
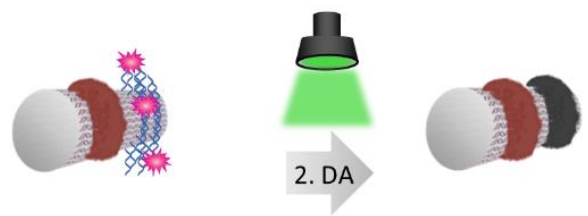

B

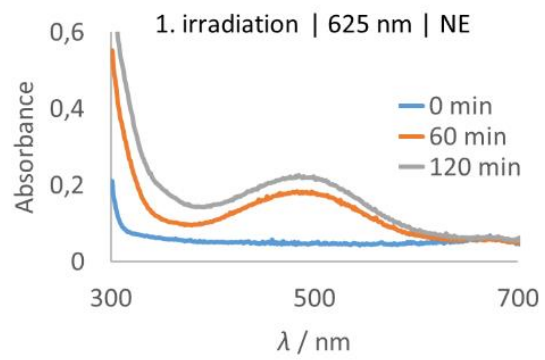

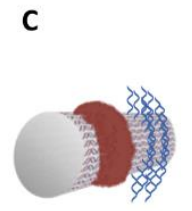

1. irradiation
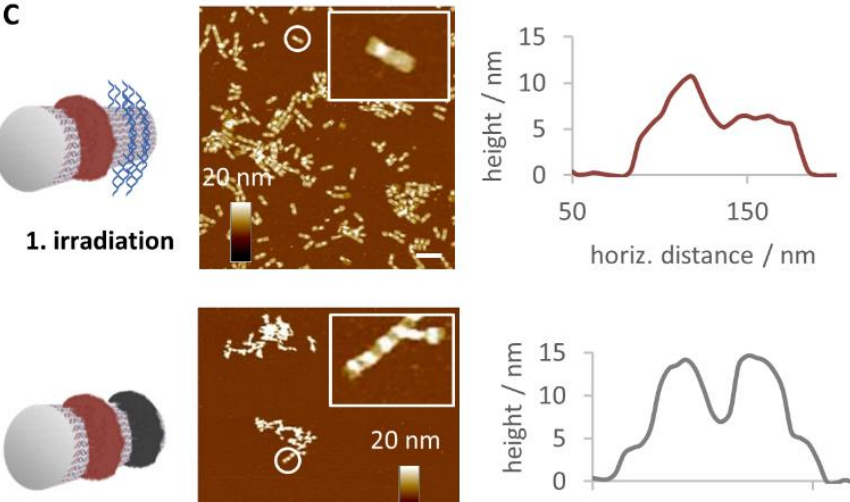

2. irradiation

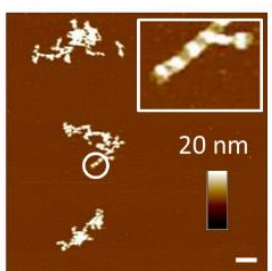

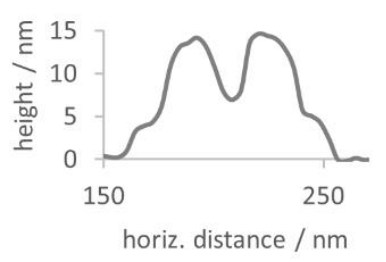

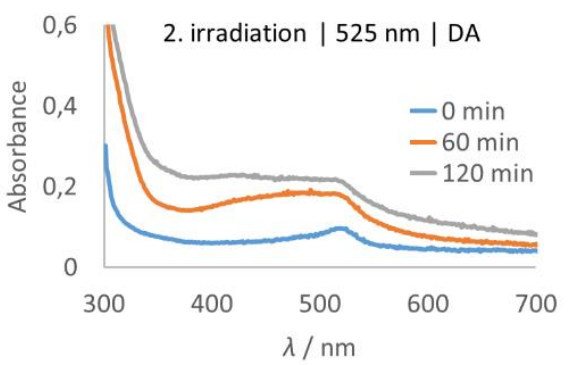

D
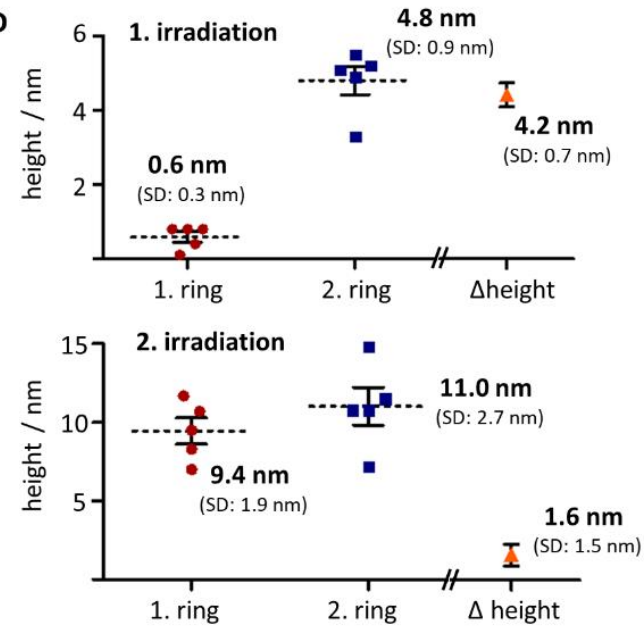

Figure 3 In an advanced two-step polymerization approach, DNA origami tubes were equipped with two different photosensitizers and incubated with two different monomers, which induced polymer formation at distinct rings at different wavelengths. (A) DNA origami tubes were endowed with one ring of G4-sequences (orange) and one ring of sticky sequences (blue). MB was loaded onto the G4 sequences, NE was added and incubated at $625 \mathrm{~nm}$. After the first irradiation phase, NE was removed by spin filtration. EY-G4 were annealed on the origami tube, and pDA formation was triggered at $525 \mathrm{~nm}$. (B) UV/Vis spectra give the characteristic profiles for pNE and pDA formation, respectively. (C) AFM imaging of origami tubes after each step was performed to track and compare the formation of the first and the second polymer ring. Scale bars are $200 \mathrm{~nm}$. Representative height profiles of one ring and two ring structures are depicted. (D) Histograms show that in the first step only one polymer ring is formed, whereas in the second step a second ring is grown. Both rings have similar heights ( $n=5$; error bars are SEM).

Importantly, the formation of these polymer patterns can be used as a tool to customize the surface chemistry of the DNA origami. Fundamentally, DNA origami structures are highly anionic due to the polyphosphate backbone and thus require divalent cations for stabilization.[17] In physiological conditions, DNA origami is easily degraded by nucleases and the lower concentration of divalent cations in biological fluids. ${ }^{[18]}$ In addition, its polyanionic character prevents cellular uptake due to repulsion against the negatively charged cellular membrane Existing strategies to address this challenge include the attachment of targeting functions (i.e., peptides, proteins, and aptamers) that promote receptor mediated endocytosis. ${ }^{[19]}$ In contrast, we hypothesized that the coverage provided by the polymer patterns would reduce the effective charge repulsion and thereby improve transport across the cellular membrane. The respective ring patterned DNA origami nanostructures with either pNE or pDA were synthesized and annealed with Alexa-647® oligonucleotide. At $5 \mathrm{nM}$, an efficient cellular uptake into 
A549 lung adenocarcinoma cells by pNE-origami was observed by confocal laser scanning microscopy after $24 \mathrm{~h}$ incubation (SI Figure 12). However, significant aggregation was observed for pDA-origami due to the well-known adhesiveness of pDA which is further enhanced under cell culture conditions. In agreement with the literature, Alexa-647® labelled DNA origami without polymers do not show uptake into cells and neither does the Alexa- $647 \AA$ oligonucleotide. The stability of the internalized pNE-origami was characterized by annealing another fluorophore Alexa-488® onto the opposite end of the tube to facilitate co-localization studies (Figure 4). By overlaying both channels, co-localization of the two fluorophores demonstrated that a major proportion of pNE-origami structures remain intact upon internalization. Similarly, controls without pNE showed no cellular uptake and the pDA-origami's aggregation propensity was likewise consistent. Through these cell experiments, we demonstrate that polymer patterns on DNA origami could be leveraged to alter and modulate interactions at the cellular interface.

A
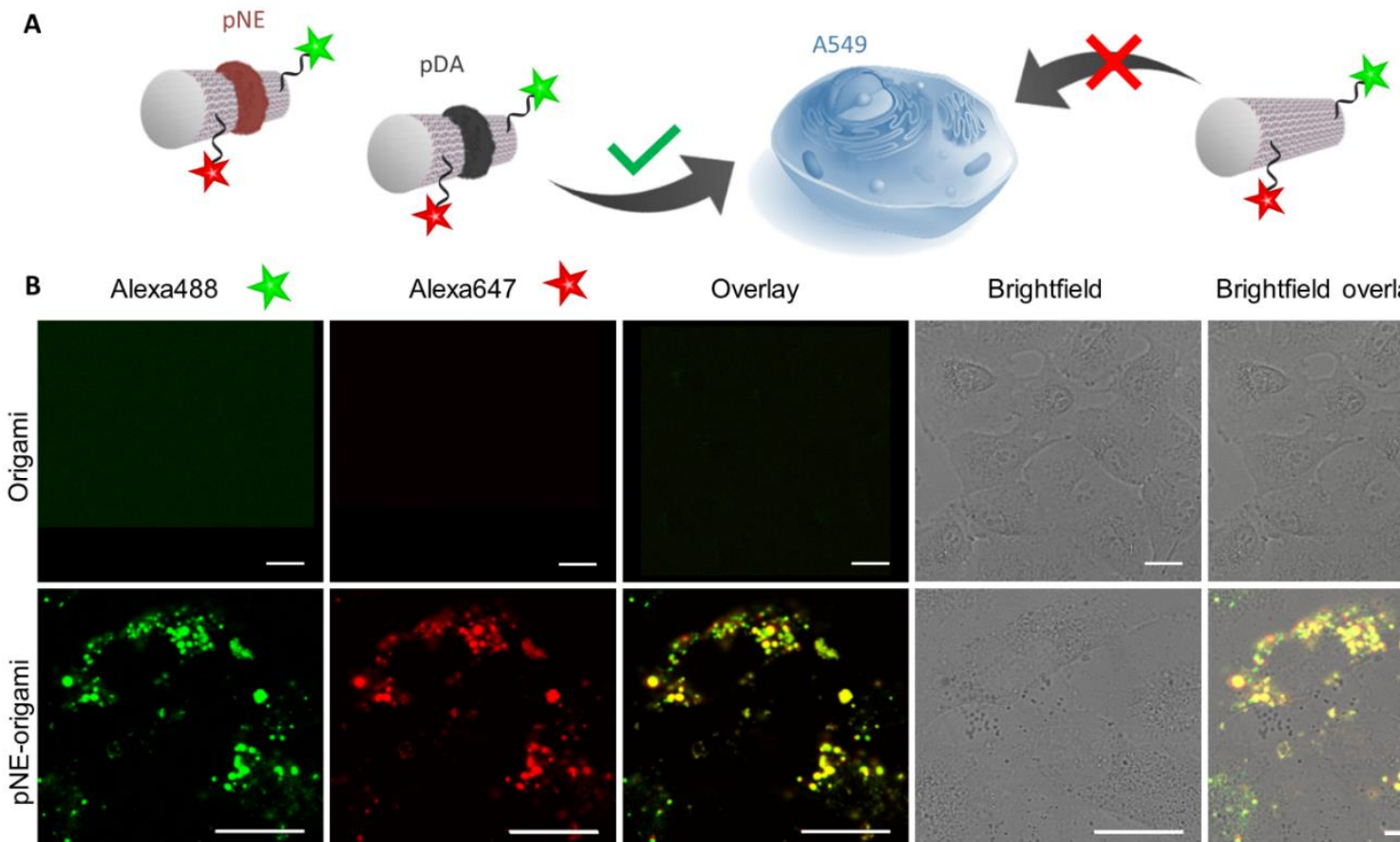

Alexa647

Overlay

Brightfield

Brightfield overlay
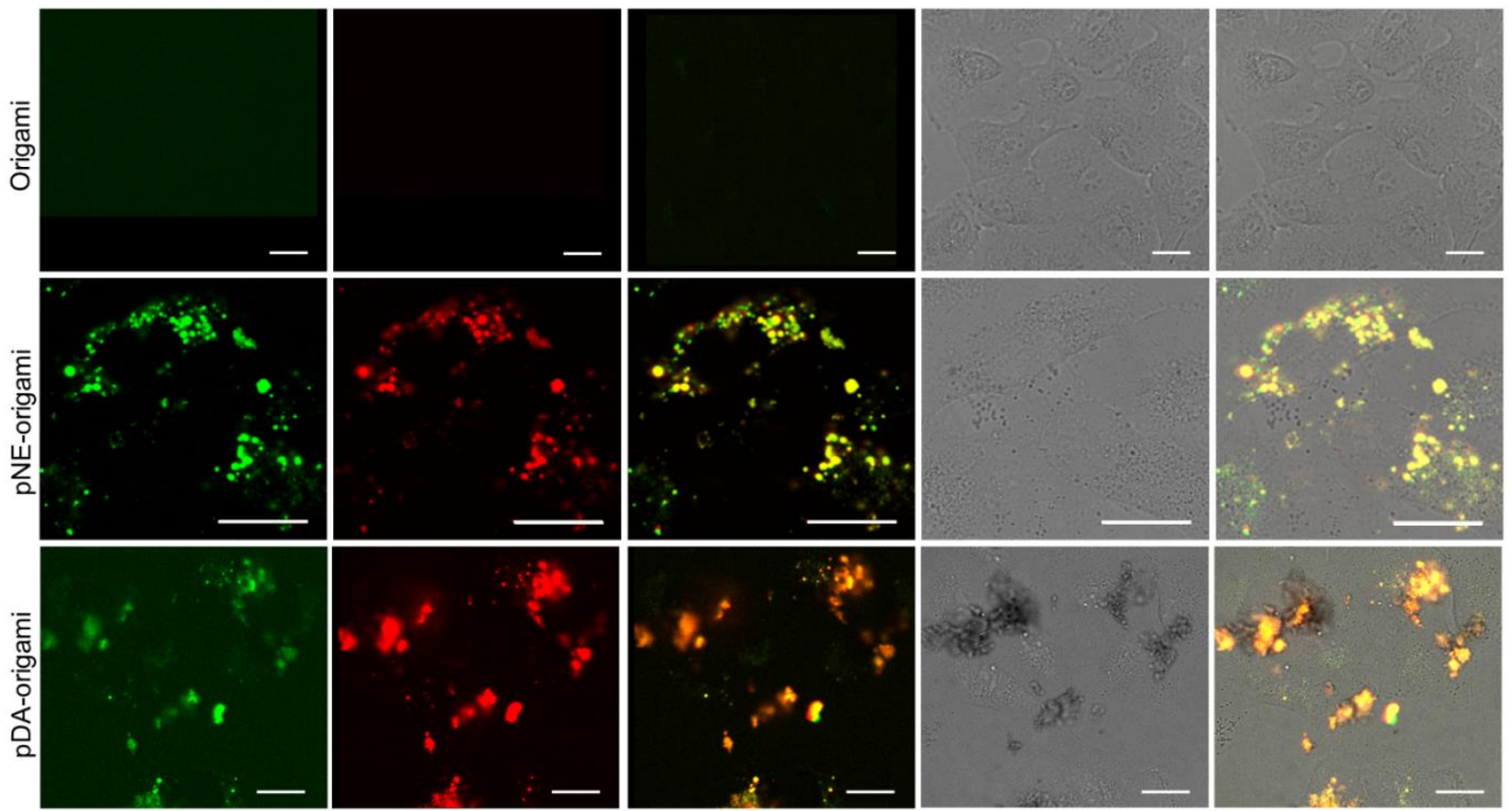

Figure 4 Cellular colocalization of origami nanostructures. (A) Bare DNA origami tubes as well as pNE- and pDA- ringed tubes were modified with both Alexa488 and Alexa647 dyes. (B) Confocal laser scanning micrographs of A549 cells treated with Alexa488 and Alexa647 double-labeled DNA origami tubes for $24 \mathrm{~h}$. Scale bars are $20 \mu \mathrm{m}$. Control images for buffer only are shown in the supporting information (SI Figure 13).

In summary, we have explored the patterned and layered growth of different polymers (pNE and pDA) on DNA origami using orthogonal wavelengths of light. The concept is facilitated by manipulating the interaction of G4s with different photosensitizers (PPIX, EY and MB) such that their position on the DNA origami can be precisely located. As a consequence, the activity of each photocatalyst can be switched from active to dormant states, and vice versa. Moreover, the fabrication method is flexible such that the sequence of the photopolymerization reaction and/or annealing steps can be changed easily without affecting their efficiencies. The extent of polymer formation can be tracked easily by UV/Vis spectroscopy and AFM imaging which facilitates structural customization in the z-direction. Furthermore, the polymer patterns altered the intrinsic polyanionic character of DNA origami while preserving their integrity. By modulating repulsive forces against the cellular membrane, these hybrid objects could be used for biological applications. In combination, this platform has provided a valuable tool to construct 
complex polymer-origami architectures that enable the study of customized surface patterns in nanoscience and biomedicine. 


\section{Acknowledgements}

The authors acknowledge the financial support by the Deutsche Forschungsgemeinschaft (DFG, German Research Foundation) - Project No. 407426226 - TRR 234 CataLight (B01) and the Max Planck-Bristol Centre for Minimal Biology.

\section{References}

[1] (a) N. C. Seeman, H. F. Sleiman, Nat. Rev. Mater. 2017, 3; (b) N. C. Seeman, J. Theor. Biol. $1982,99,237-247$.

[2] P. W. Rothemund, Nature 2006, 440, 297-302.

[3] (a) P. Zhang, X. Liu, P. Liu, F. Wang, H. Ariyama, T. Ando, J. Lin, L. Wang, J. Hu, B. Li, C. Fan, Nat. Commun. 2020, 11, 3114; (b)J. Hellmeier, R. Platzer, A. S. Eklund, T. Schlichthaerle, A. Karner, V. Motsch, M. C. Schneider, E. Kurz, V. Bamieh, M. Brameshuber, J. Preiner, R. Jungmann, H. Stockinger, G. J. Schutz, J. B. Huppa, E. Sevcsik, PNAS 2021, 118, DOI: 10.1073/pnas.2016857118.

[4] (a) G. Grossi, M. Dalgaard Ebbesen Jepsen, J. Kjems, E. S. Andersen, Nat. Commun. 2017, 8, 992; (b) W. P. Klein, R. P. Thomsen, K. B. Turner, S. A. Walper, J. Vranish, J. Kjems, M. G. Ancona, I. L. Medintz, ACS Nano 2019, 13, 13677-13689; (c) E. Weinhold, B. Chakraborty, Nanoscale 2021, 13, 2465-2471.

[5] (a) J. Ryssy, A. K. Natarajan, J. Wang, A. J. Lehtonen, M. K. Nguyen, R. Klajn, A. Kuzyk, Angew. Chem. Int. Ed. 2021, 60, 5859-5863; (b) L. Nguyen, M. Dass, M. F. Ober, L. V. Besteiro, Z. M. Wang, B. Nickel, A. O. Govorov, T. Liedl, A. Heuer-Jungemann, ACS Nano 2020, 14, 7454-7461.

[6] (a) E. Silvester, B. Vollmer, V. Prazak, D. Vasishtan, E. A. Machala, C. Whittle, S. Black, J. Bath, A. J. Turberfield, K. Grunewald, L. A. Baker, Cell 2021, 184, 1110-1121; (b) J. Huang, A. Suma, M. Cui, G. Grundmeier, V. Carnevale, Y. Zhang, C. Kielar, A. Keller, Small Struct. 2020, 1, DOI: 10.1002/sstr.202000038.

[7] (a) Z.-G. Wang, Q. Liu, B. Ding, Chem. Mater. 2014, 26, 3364-3367; (b) Y. Tokura, Y. Jiang, A. Welle, M. H. Stenzel, K. M. Krzemien, J. Michaelis, R. Berger, C. Barner-Kowollik, Y. Wu, T. Weil, Angew. Chem. Int. Ed. 2016, 55, 5692-5697; (c) Y. Tokura, S. Harvey, C. Chen, Y. Wu, D. Y. W. Ng, T. Weil, Angew. Chem. Int. Ed. 2018, 57, 1587-1591; (d) J. B. Knudsen, L. Liu, A. L. Bank Kodal, M. Madsen, Q. Li, J. Song, J. B. Woehrstein, S. F. Wickham, M. T. Strauss, F. Schueder, J. Vinther, A. Krissanaprasit, D. Gudnason, A. A. Smith, R. Ogaki, A. N. Zelikin, F. Besenbacher, V. Birkedal, P. Yin, W. M. Shih, R. Jungmann, M. Dong, K. V. Gothelf, Nat. Nanotechnol. 2015, 10, 892-898; (e) A. Krissanaprasit, M. Madsen, J. B. Knudsen, D. Gudnason, W. Surareungchai, V. Birkedal, K. V. Gothelf, ACS Nano 2016, 10, 2243-2250.

[8] (a) P. Chidchob, T. G. Edwardson, C. J. Serpell, H. F. Sleiman, JACS 2016, 138, 4416-4425; (b) A. Lacroix, T. G. W. Edwardson, M. A. Hancock, M. D. Dore, H. F. Sleiman, JACS 2017, 139, 73557362; (c) Y. Dong, Y. R. Yang, Y. Zhang, D. Wang, X. Wei, S. Banerjee, Y. Liu, Z. Yang, H. Yan, D. Liu, Angew. Chem. Int. Ed. 2017, 56, 1586-1589; (d) J. A. Fan, Y. He, K. Bao, C. Wu, J. Bao, N. B. Schade, V. N. Manoharan, G. Shvets, P. Nordlander, D. R. Liu, F. Capasso, Nano Lett. 2011, 11, 4859-4864; (e) L. Song, Q. Jiang, J. Liu, N. Li, Q. Liu, L. Dai, Y. Gao, W. Liu, D. Liu, B. Ding, Nanoscale 2017, 9, $7750-$ 7754; (f) W. Chen, J. Y. Gerasimov, P. Zhao, K. Liu, A. Herrmann, JACS 2015, 137, 12884-12889; (g) G. Chakraborty, K. Balinin, G. Portale, M. Loznik, E. Polushkin, T. Weil, A. Herrmann, Chem. Sci. 2019, 10, 10097-10105; (h) H. Li, J. Fan, E. M. Buhl, S. Huo, M. Loznik, R. Gostl, A. Herrmann, Nanoscale 2020, 12, 21299-21305; (i) Y. Yang, Q. Lu, C. M. Huang, H. Qian, Y. Zhang, S. Deshpande, G. Arya, Y. Ke, S. Zauscher, Angew. Chem. Int. Ed. 2021, DOI: 10.1002/anie.202107829.

[9] (a) P. Wang, J. H. Huh, H. Park, D. Yang, Y. Zhang, Y. Zhang, J. Lee, S. Lee, Y. Ke, Nano Lett. 2020, 20, 8926-8932; (b) K. Zhou, Y. Zhou, V. Pan, Q. Wang, Y. Ke, JACS 2020, 142, 5929-5932.

[10] P. Winterwerber, S. Harvey, D. Y. W. Ng, T. Weil, Angew. Chem. Int. Ed. 2020, 59, 6144-6149. 
[11] (a) S. Hong, Y. S. Na, S. Choi, I. T. Song, W. Y. Kim, H. Lee, Adv. Funct. Mater. 2012, 22, 47114717; (b) D. R. Dreyer, D. J. Miller, B. D. Freeman, D. R. Paul, C. W. Bielawski, Langmuir 2012, 28, 6428-6435; (c) H. Lee, S. M. Dellatore, W. M. Miller, P. B. Messersmith, Science 2007, 318, 426-430.

[12] (a) P. Manini, A. Pezzella, L. Panzella, A. Napolitano, M. d'Ischia, Tetrahedron 2005, 61, 40754080; (b) P. Manini, L. Panzella, A. Napolitano, M. d'Ischia, Chem. Res. Toxicol. 2007, 20, 1549-1555; (c) S. Hong, J. Kim, Y. S. Na, J. Park, S. Kim, K. Singha, G. I. Im, D. K. Han, W. J. Kim, H. Lee, Angew. Chem. Int. Ed. 2013, 52, 9187-9191; (d) X. Tan, P. Gao, Y. Li, P. Qi, J. Liu, R. Shen, L. Wang, N. Huang, K. Xiong, W. Tian, Q. Tu, Bioact. Mater. 2021, 6, 285-296.

[13] (a) F. Amat-Guerri, M. M. C. López-González, R. Martínez-Utrilla, R. Sastre, J. Photochem. Photobiol. 1990, 53, 199-210; (b) D. P. Hari, B. Konig, Chem. Commun. 2014, 50, 6688-6699; (c) V. Srivastava, P. P. Singh, RSC Adv. 2017, 7, 31377-31392.

[14] (a) J. P. Tardivo, A. Del Giglio, C. S. de Oliveira, D. S. Gabrielli, H. C. Junqueira, D. B. Tada, D. Severino, R. de Fátima Turchiello, M. S. Baptista, Photodiagn. Photodyn. Ther. 2005, 2, 175-191; (b) P. T. Wu, C. L. Lin, C. W. Lin, N. C. Chang, W. B. Tsai, J. Yu, Nanomaterials 2018, 9, 14.

[15] I. Kraljić, S. E. Mohsni, Photochem. Photobiol. 1978, 28, 577-581.

[16] (a) S. M. Kang, J. Rho, I. S. Choi, P. B. Messersmith, H. Lee, JACS 2009, 131, 13224-13225; (b) H. A. Lee, Y. Ma, F. Zhou, S. Hong, H. Lee, Acc. Chem. Res. 2019, 52, 704-713.

[17] (a) C. Kielar, Y. Xin, B. Shen, M. A. Kostiainen, G. Grundmeier, V. Linko, A. Keller, Angew. Chem. Int. Ed. 2018, 57, 9470-9474; (b) J. A. L. Roodhuizen, P. Hendrikx, P. A. J. Hilbers, T. F. A. de Greef, A. J. Markvoort, ACS Nano 2019, 13, 10798-10809.

[18] (a) J. Hahn, S. F. Wickham, W. M. Shih, S. D. Perrault, ACS Nano 2014, 8, 8765-8775; (b) H. Bila, E. E. Kurisinkal, M. M. C. Bastings, Biomater. Sci. 2019, 7, 532-541.

[19] (a) S. Ko, H. Liu, Y. Chen, C. Mao, Biomacromolecules 2008, 9, 3039-3043; (b) S. D. Perrault, W. M. Shih, ACS Nano 2014, 8, 5132-5140; (c) M. M. C. Bastings, F. M. Anastassacos, N. Ponnuswamy, F. G. Leifer, G. Cuneo, C. Lin, D. E. Ingber, J. H. Ryu, W. M. Shih, Nano Lett. 2018, 18, 3557-3564; (d) S. Li, Q. Jiang, S. Liu, Y. Zhang, Y. Tian, C. Song, J. Wang, Y. Zou, G. J. Anderson, J. Y. Han, Y. Chang, Y. Liu, C. Zhang, L. Chen, G. Zhou, G. Nie, H. Yan, B. Ding, Y. Zhao, Nat. Biotechnol. 2018, 36, 258264; (e) J. Liu, L. Song, S. Liu, Q. Jiang, Q. Liu, N. Li, Z. G. Wang, B. Ding, Nano Lett. 2018, 18, $3328-$ 3334; (f) Q. Pan, C. Nie, Y. Hu, J. Yi, C. Liu, J. Zhang, M. He, M. He, T. Chen, X. Chu, ACS Appl. Mater. Interfaces 2020, 12, 400-409. 